\title{
藤黄酸重排反应产物研究
}

\author{
杨 金金孙中强双云霞郡志宇* \\ (东华大学化学化工与生物工程学院 上海 201620)
}

\begin{abstract}
摘要 藤黄酸能显著抑制多种人类肿瘤细胞的增殖，在酸性环境中易发生重排反应，其重排产物对肿瘤细胞也具有很 强的抑制作用. 通过波谱技术确定了两个藤黄酸的重排反应产物的结构并归属了其波谱数据. 细胞毒活性测试显示, 两个重排产物对 A549, HCT116 和 MDA-MB-231 显示很强的抑制作用.
\end{abstract}

关键词 藤黄酸; 重排反应; 波谱技术; 细胞毒活性

\section{Research of Rearrangement Products of Gambogic Acid}

\author{
Yang, Xin Sun, Zhongqiang Deng, Yunxia Shao, Zhiyu* \\ (College of Chemistry, Chemical Engineering and Biotechnology, Donghua University, Shanghai 201620)
}

\begin{abstract}
Gambogic acid derivatives possess potent anti-tumor activities against various cancer cell lines. In a search for new anti-tumor organic compounds, rearrangement reaction of gambogic acid was explored. After microwave irradiation under acidic condition, two rearrangement products were isolated and their structures were determined unambiguously by spectroscopic techniques. Both of them were screened for their cytotoxicities against A549, HCT116 and MDA-MB-231, and showed high inhibitory effects on the cell lines.
\end{abstract}

Keywords gambogic acid; rearrangement reaction; spectroscopy; cytotoxicity

藤黄(gamboge) 是藤黄树(Garcinia hanburyi Hook. f.)分泌的树脂, 藤黄酸(1)为藤黄的主要有效成分 ${ }^{[1]}$. 藤 黄酸生物合成来源于混合的乙酰源和莽草酸生源, 结构 复杂, 具有呫吨酮 (xanthone) 和独特的 4-氧杂三环 [4.3.1. $0^{3,7}$ 癸-2-酮笼状骨架. 生物活性研究表明 ${ }^{[2 ~ 5]}$, 藤 黄酸能显著抑制多种人类肿瘤细胞的增殖: 如人白血病 K562 细胞、人肝癌 SMMC-7721 细胞株和胃腺癌细胞株 SGC-7901, 而且在体内体外实验中藤黄酸均不影响正 常细胞. 因此对藤黄酸的结构改造工作早已展开, 早先 的研究报道表明藤黄酸的 A 环在酸性环境下容易发生 重排, 产生复杂多变的新颖结构, 其重排衍生物大多显 示高于前体的细胞毒活性. 冯峰等 ${ }^{[6]}$ 合成的 33 -氯化转 位藤黄酸和 33,37 -二氯转位藤黄酸对人肝细胞性肝癌 (SMMC-7721)细胞生长活性的抑制作用较藤黄酸更强. 为了寻找新抗癌化合物, 从而进行藤黄酸重排反应的研 究, 希望可以发现具有良好抗癌活性的化合物. 本文以 醋酸为溶剂, 对甲苯磺酸为质子酸, 在加热或微波照射 下获得藤黄酸重排反应产物 2 和 3 (Eq. 1). 我们对其结
构进行了波谱鉴定, 其中一个为新衍生物 3 , 另一个化 合物 2 虽已报道, 但是波谱数据差异较大. 两个化合物 的细胞毒活性测试显示对人肺癌细胞 A549、人结肠癌 细胞 HCT116 和人乳腺癌细胞 MDA-MB-231 有显著抑 制.

\section{1 结果与讨论}

\section{1 波谱分析}

化合物 2: 黄色的粉末状固体，由 HR-ESI-MS $\{\mathrm{m} / \mathrm{z}$ $629.31089[\mathrm{M}+\mathrm{H}]^{+}$, calcd 629.31144$\}$ 给出分子式为 $\mathrm{C}_{38} \mathrm{H}_{44} \mathrm{O}_{8}$, 不饱和度为 16 , 与藤黄酸相同. ${ }^{1} \mathrm{H} N M R$ 中给 出 $\mathbf{2}$ 的类藤黄酸的特征性信号, 一个分子内氢键化的羟 基 $[\delta 12.71(\mathrm{~s}, 1 \mathrm{H}, 6-\mathrm{OH})]$ ，三个烯质子 $[7.50(\mathrm{~d}, J=6.8$ $\mathrm{Hz}, 1 \mathrm{H}, \mathrm{H}-10), 6.03$ (t, $J=7.3 \mathrm{~Hz}, 1 \mathrm{H}, \mathrm{H}-27)$ 和 5.05 (t, $J=$ $5.6 \mathrm{~Hz}, 1 \mathrm{H}, \mathrm{H}-32)]$ ，八个甲基 $[\delta 1.84,1.74,1.73,1.71$ ， $1.64,1.61,1.32$ 和 1.29$]$. 将化合物 $\mathbf{2}$ 的全部 ${ }^{1} \mathrm{H}$ NMR 和 ${ }^{13} \mathrm{C} \mathrm{NMR}$ 数据(表 1)与藤黄酸比较, 2 与藤黄酸整体骨架

\footnotetext{
*E-mail: zyshao@dhu.edu.cn.

Received October 28, 2014; revised November 23, 2014; published online December 9, 2014.

Project supported by the Fundamental Research Funds for the Central Universities (Nos. 13D110513, 2232012D3-03).

中央高校基本科研业务费专项资金(Nos. 13D110513, 2232012D3-03)资助项目.
} 

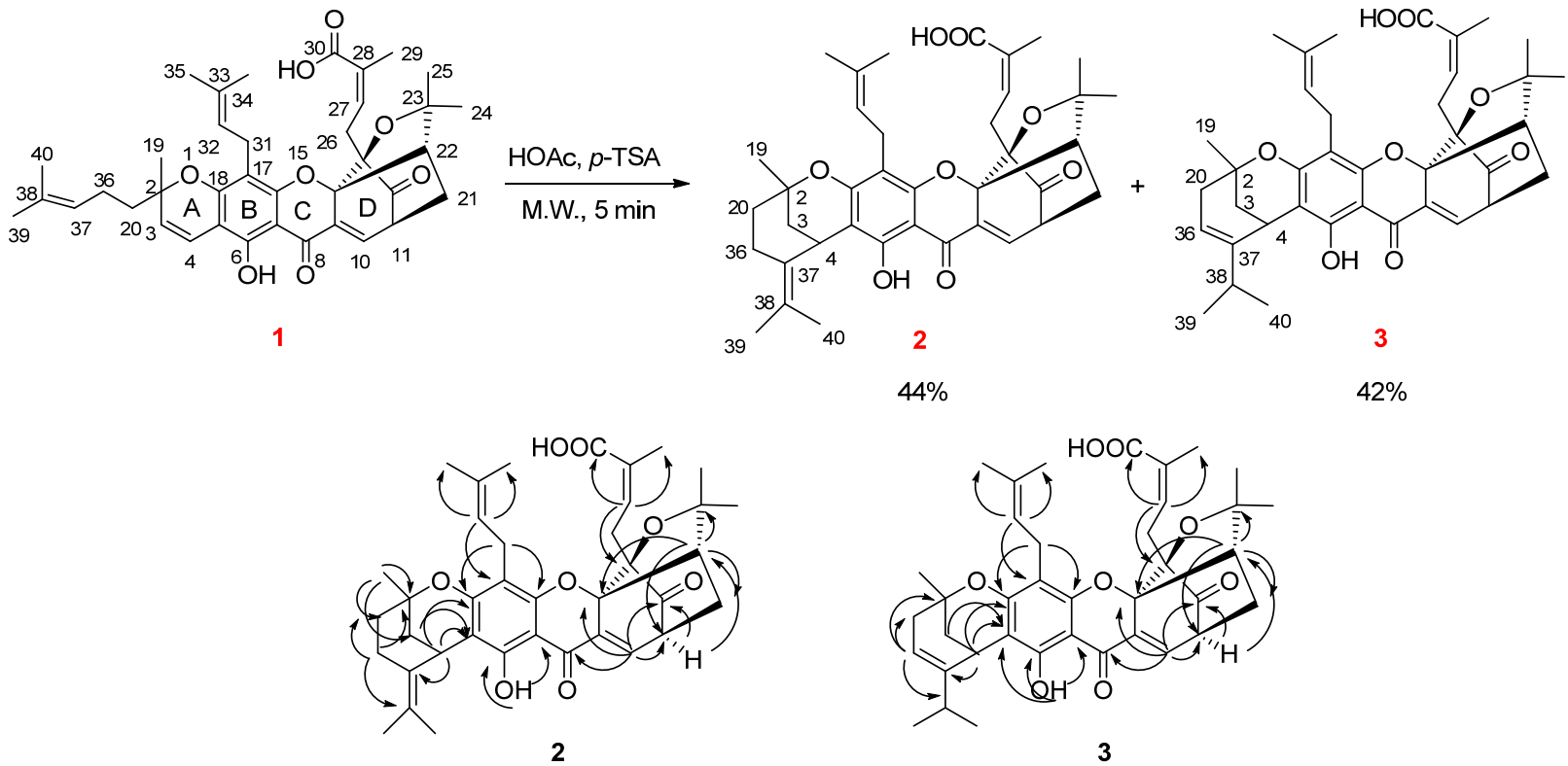

图 1 化合物 $\mathbf{2}$ 和 3 的主要 $\mathrm{HMBC}(\mathrm{H} \rightarrow \mathrm{C})$ 相关

Figure 1 The main HMBC $(\mathrm{H} \rightarrow \mathrm{C})$ correlations of compounds $\mathbf{2}$ and $\mathbf{3}$

表 1 化合物 2 和 3 的 1D-NMR 数据

Table 1 1D-NMR data of compounds $\mathbf{2}$ and $\mathbf{3}$

\begin{tabular}{|c|c|c|c|c|c|c|c|c|c|}
\hline \multirow{2}{*}{ Position } & \multicolumn{2}{|l|}{2} & \multicolumn{2}{|l|}{3} & \multicolumn{3}{|c|}{2} & \multicolumn{2}{|l|}{3} \\
\hline & $\delta_{\mathrm{H}}$ & $\delta_{\mathrm{C}}$ & $\delta_{\mathrm{H}}$ & $\delta_{\mathrm{C}}$ & Position & $\delta_{\mathrm{H}}$ & $\delta_{\mathrm{C}}$ & $\delta_{\mathrm{H}}$ & $\delta_{\mathrm{C}}$ \\
\hline 2 & & 77.25 & & 77.25 & 24 & $1.71(\mathrm{~s}, 3 \mathrm{H})$ & 29.90 & $1.70(\mathrm{~s}, 3 \mathrm{H})$ & 29.89 \\
\hline 3 & $1.72(\mathrm{~m}, 2 \mathrm{H})$ & 36.21 & $\begin{array}{l}1.86(\mathrm{dd}, 1 \mathrm{H}, 12.5, \\
3.0), 1.72(\mathrm{~m}, 1 \mathrm{H})\end{array}$ & 34.11 & 25 & $1.29(\mathrm{~s}, 3 \mathrm{H})$ & 28.91 & $1.10(\mathrm{~s}, 3 \mathrm{H})$ & 28.87 \\
\hline 4 & $4.15(\mathrm{~s}, 1 \mathrm{H})$ & 28.72 & $3.75(\mathrm{~s}, 1 \mathrm{H})$ & 27.00 & 26 & $3.02(\mathrm{~d}, 2 \mathrm{H}, 7.3)$ & 29.19 & $2.95(\mathrm{t}, 2 \mathrm{H}, 7.5)$ & 29.29 \\
\hline 5 & & 106.88 & & 108.02 & 27 & $6.03(\mathrm{t}, 1 \mathrm{H}, 7.3)$ & 138.34 & $6.04(\mathrm{t}, 1 \mathrm{H}, 7.5)$ & 137.70 \\
\hline 6 & & 159.41 & & $158.61 \|$ & 28 & & 127.78 & & 127.90 \\
\hline 7 & & 100.01 & & 99.90 & 29 & $1.73(\mathrm{~s}, 3 \mathrm{H})$ & 20.73 & $1.73(\mathrm{~s}, 3 \mathrm{H})$ & 20.78 \\
\hline 8 & & 178.61 & & 178.65 & 30 & & 171.58 & & 170.73 \\
\hline 9 & & 133.97 & & 133.84 & 31 & $\begin{array}{l}3.29(\mathrm{dd}, 1 \mathrm{H}, 14.2 \\
8.2), 3.10(\mathrm{dd}, 1 \mathrm{H} \\
14.4,4.7)\end{array}$ & 21.83 & $\begin{array}{l}3.27(\mathrm{dd}, 1 \mathrm{H}, 14.5 \\
8.0), 3.11(\mathrm{dd}, 1 \mathrm{H}, \\
14.5,5.5)\end{array}$ & 21.92 \\
\hline 10 & $7.50(\mathrm{~d}, 1 \mathrm{H}, 6.8)$ & 134.46 & $7.52(\mathrm{~d}, 1 \mathrm{H}, 7.0)$ & 134.62 & 32 & $5.05(\mathrm{t}, 1 \mathrm{H}, 5.6)$ & 122.47 & $5.07(\mathrm{t}, 1 \mathrm{H}, 6.5)$ & 122.47 \\
\hline 11 & $3.45(\mathrm{t}, 1 \mathrm{H}, 5.8)$ & 46.75 & $3.46(\mathrm{t}, 1 \mathrm{H}, 5.5)$ & 46.08 & 33 & & 131.07 & & 131.16 \\
\hline 12 & & 203.62 & & 203.54 & 34 & $1.74(\mathrm{~s}, 3 \mathrm{H})$ & 18.15 & $1.73(\mathrm{~s}, 3 \mathrm{H})$ & 18.15 \\
\hline 13 & & 84.28 & & 84.11 & 35 & $1.64(\mathrm{~s}, 3 \mathrm{H})$ & 25.77 & $1.64(\mathrm{~s}, 3 \mathrm{H})$ & 25.76 \\
\hline 14 & & 90.46 & & 90.46 & 36 & $\begin{array}{l}2.39(\mathrm{dd}, 1 \mathrm{H}, 14.5,5) \\
1.69(\mathrm{~m}, 1 \mathrm{H})\end{array}$ & 22.61 & $5.17(\mathrm{~s}, 1 \mathrm{H})$ & 114.16 \\
\hline 16 & & 155.14 & & 155.31 & 37 & & 130.49 & & 149.18 \\
\hline 17 & & 106.65 & & 107.42 & 38 & & 122.62 & $2.44(\mathrm{~m}, 1 \mathrm{H})$ & 31.65 \\
\hline 18 & & 164.01 & & 162.77 & 39 & $1.61(\mathrm{~s}, 3 \mathrm{H})$ & 20.65 & $1.07(\mathrm{~d}, 3 \mathrm{H}, 7.0)$ & 22.20 \\
\hline 19 & $1.32(\mathrm{~s}, 3 \mathrm{H})$ & 28.38 & $1.50(\mathrm{~s}, 3 \mathrm{H})$ & 28.72 & 40 & $1.84(\mathrm{~s}, 3 \mathrm{H})$ & 20.22 & $0.92(\mathrm{~d}, 3 \mathrm{H}, 7.0)$ & 20.51 \\
\hline 20 & $\begin{array}{l}1.89(\mathrm{~m}, 1 \mathrm{H}) \\
1.48(\mathrm{~m}, 1 \mathrm{H})\end{array}$ & 40.09 & $2.33(2 \mathrm{H}, \mathrm{m})$ & 40.32 & 42 & & & & \\
\hline 21 & $\begin{array}{l}2.30(\mathrm{dd}, 1 \mathrm{H}, 13.5 \\
4.5), 1.38(\mathrm{~m}, 1 \mathrm{H})\end{array}$ & 25.25 & $\begin{array}{l}2.28(\mathrm{~m}, 1 \mathrm{H}) \\
1.35(\mathrm{~m}, 1 \mathrm{H})\end{array}$ & 25.20 & 43 & & & & \\
\hline 22 & $2.48(\mathrm{~d}, 1 \mathrm{H}, 9.5)$ & 49.01 & $2.46(\mathrm{~d}, 1 \mathrm{H}, 9.5)$ & 49.02 & $6-\mathrm{OH}$ & $12.71(\mathrm{~s}, 1 \mathrm{H})$ & & $12.81(\mathrm{~s}, 1 \mathrm{H})$ & \\
\hline 23 & & 83.90 & & 83.99 & & & & & \\
\hline
\end{tabular}


类似, xanthone 结构的 B、C 和 D 环结构没有变化, 而前 体中的 A 环的联接基团发生了变化. A 环中 C-3 和 C-4 之间双键消失. ${ }^{1} \mathrm{H}$ NMR 谱中一个特征的信号 $\delta 4.15$ (brs, 1H) 在 HSQC 中显示与信号 $\delta$ 为 28.72 的碳原子相 连, 在 $\mathrm{HMBC}$ (图 1)中分别与 $\delta 164.01$ (C-18), 159.41 (C-6), 130.49 (C-37), 122.62 (C-38), 106.88 (C-5), 77.25 (C-2), 36.21 (C-3), 22.61 (C-36)相关, 由此分析 $\delta 4.15$ 是 C-4 上连接的氢信号. 前体藤黄酸中的 H-37 信号在 2 中 消失, 分析 2 中 $\mathrm{sp}^{2}$ 杂化碳信号发现比藤黄酸少 2 个, 由 于不饱和度与藤黄酸相同, 由此推出分子中多出一个 环. ${ }^{13} \mathrm{C}$ NMR 中 $\delta 77.25(\mathrm{C}-2)$ 分别与 $\delta 4.15(\mathrm{H}-4), 2.39$ (Ha-36), 1.89 (Ha-20), 1.61 (H-3), 1.48 (Hb-20), 1.32 (H-19)相关, 再次验证化合物 2 中新形成的六元环, 综 合数据得到 $\mathbf{2}$ 的结构.

化合物 3: 黄色的粉末状固体, 由 HR-ESI-MS $(\mathrm{m} / \mathrm{z}$ $629.31089[\mathrm{M}+\mathrm{H}]^{+}$, calcd 629.31144)确定分子式为 $\mathrm{C}_{38} \mathrm{H}_{44} \mathrm{O}_{8}$, 不饱和度为 $16 .{ }^{1} \mathrm{H}$ NMR 给出了 3 的特征性信 号, 一个形成分子内氢键的羟基 $[\delta 12.81(\mathrm{~s}, 1 \mathrm{H}, 6-\mathrm{OH})]$, 四个烯质子 $[\delta 7.52(\mathrm{~d}, J=7.0 \mathrm{~Hz}, 1 \mathrm{H}, \mathrm{H}-10), 6.04(\mathrm{t}, J=$ $7.5 \mathrm{~Hz}, 1 \mathrm{H}, \mathrm{H}-27), 5.17$ (s, 1H, H-36) 和 5.07 (t, $J=6.5 \mathrm{~Hz}$, $1 \mathrm{H}, \mathrm{H}-32)]$ ，八个甲基 $[\delta 1.73,1.73,1.70,1.64,1.50,1.10$, 1.07 和 0.92$]$; 将 3 的全部 ${ }^{1} \mathrm{H} \mathrm{NMR}$ 和 ${ }^{13} \mathrm{C}$ NMR 数据(表 1)与 $\mathbf{2}$ 比较发现, 3 与 $\mathbf{2}$ 整体骨架相同, 其仅有的区别在 于 2 中的 C-37 和 C-38 之间的双键在 $\mathbf{3}$ 中转移到 C-36 和 C-37 位. 通过 ${ }^{1} \mathrm{H} N M R 、{ }^{13} \mathrm{C}$ NMR、2D-NMR (HSQC、 $\mathrm{HMBC}$ )分析可知, 在 HSQC 中 $\delta 22.20$ (C-39)、20.51 (C-40)分别对应 $\delta 1.07(\mathrm{H}-39), 0.92(\mathrm{H}-40)$, 在 ${ }^{1} \mathrm{H}$ NMR 中这两个峰均成 $\mathrm{d}$ 峰, 说明 C-37 和 C-38 位双键消失, 此 位置为异丙基结构, C-39、C-40 位氢被 C-38 位氢裂分成 双重峰; ${ }^{1} \mathrm{H}$ NMR 中的 $\delta 5.17(\mathrm{H}-36)$, 在 HSQC 中与 114.16 (C-36)相关, 在 $\mathrm{HMBC}$ (图 1)中分别与 $\delta 77.25$ (C-2), 40.32 (C-20), 31.65 (C-38), 27.00 (C-4)相关, 所以 推断 2 中的 C-37 和 C-38 之间的双键在 3 中转移到 C-36 和 C-37 之间; ${ }^{13} \mathrm{C}$ NMR 中 $\delta 77.25$ (C-2)分别与 $\delta 5.17$ (H-36), 3.75 (H-4), 2.33 (H-20), 1.71 (Hb-3), 1.50 (H-19) 相关, 且在碳谱中化学位移达到 $\delta 77.25$, 所以判定在 C-2 和 C-18 之间有一个氧原子, 这个推断也被 C-18 化 学位移 $\delta 162.77$ 和 ESI 准分子离子峰([M+H $]^{+}, m / z$ 629) 所佐证. 由以上数据推出化合物 3 的结构.

\section{2 藤黄酸的重排}

以藤黄酸为起始原料, 冰醋酸中加热条件下研究其 反应产物. 经薄层层析跟踪反应发现, 在该条件下, 未 生成任何新的重排产物. 在溶剂中加入相对藤黄酸 14 equiv. 对甲苯磺酸, $80{ }^{\circ} \mathrm{C}$ 反应 $1 \mathrm{~h}$ 后, 经薄层层析检测发 现有一明显的新点, 其极性较藤黄酸略小 $[V$ (石油醚) :
$V($ 乙酸乙酯 $): V($ 甲酸 $\left.)=2: 1: 0.05, R_{\mathrm{f}}=0.68\right]$. 藤黄酸 $\left[V\left(\right.\right.$ 石油梄) $: V\left(\right.$ 乙酸乙酯) $: V($ 甲酸 $)=2: 1: 0.05, R_{\mathrm{f}}=$ $0.58]$ 点已经消失. 经 ${ }^{1} \mathrm{H}$ NMR 检测, 产物为混合物, 在 6-OH $(\delta 12 \sim 13)$ 和 C-9 和 C-10 之间的双键处 $(\delta 7.5$ 附近) 存在多重峰, 将此混合物用 HPLC 检测和分离, 最终选 用 $V$ (乙腈) $: V$ (水) $: V(0.08 \%$ 三氟乙酸 $)=80: 18: 2$, 流 速 $3 \mathrm{~mL} / \mathrm{min}$, 紫外检测波长 $254 \mathrm{~nm}$ 条件, 分离得到 2 个主要化合物 $\mathbf{2}$ 和 3 , 保留时间分别为 78 和 $63.5 \mathrm{~min}$, 产 率 $21 \%$ 和 $17 \%$.

当保持以上反应溶剂和温度不变, 增加反应时间, 继续研究该重排反应. 经薄层层析监控得, 当反应时间 为 $3 \mathrm{~h}$ 以内时, 在此条件下化合物 $\mathbf{2}$ 和 3 的混合物点仍 然存在. 将反应时间进一步增加, 薄层层析监控发现, 时间的增加, 化合物将转变成其他化合物.

藤黄酸在微波条件下的酸性溶液中也能发生重排 反应 ${ }^{[7]}$, 藤黄酸溶于冰醋酸中, 加入对甲苯磺酸, 在 $80{ }^{\circ} \mathrm{C}$ 条件下, 微波条件下反应 $5 \mathrm{~min}$. 反应产物经 HPLC 检测发现, 该条件下的反应产物与上述 $80{ }^{\circ} \mathrm{C}$ 反 应 $1 \mathrm{~h}$ 的反应产物相同, 主要产物为 $\mathbf{2}$ 和 $\mathbf{3}$, 产率显著提 高, 分别为 $44 \%$ 和 $42 \%$.

\section{3 细胞毒活性}

我们检测了化合物 $\mathbf{2}$ 和 3 对人肺癌细胞 A549, 人结 肠癌细胞 HCT116 和人乳腺癌细胞 MDA-MB-231 的细 胞毒活性, 结果见表 2.

表 2 化合物 2 和 3 对 A549, HCT116 和 MDA-MB-231 的细 胞毒活性

Table 2 Cytotoxicity of compounds $\mathbf{2}$ and $\mathbf{3}$ against A549, HCT116 and MDA-MB-231 cells

\begin{tabular}{clll}
\hline \multirow{2}{*}{ Compound } & \multicolumn{3}{c}{$\mathrm{IC}_{50}{ }^{a} /\left(\mu \mathrm{mol} \bullet \mathrm{L}^{-1}\right)$} \\
\cline { 2 - 4 } & \multicolumn{1}{c}{$\mathrm{A} 549$} & \multicolumn{1}{c}{$\mathrm{HCT} 116$} & $\mathrm{MDA}-\mathrm{MB}-231$ \\
\hline $\mathbf{2}$ & $0.55 \pm 0.035$ & $0.54 \pm 0.035$ & $0.23 \pm 0.012$ \\
$\mathbf{3}$ & $0.72 \pm 0.033$ & $1.21 \pm 0.091$ & $0.28 \pm 0.014$ \\
$\mathbf{1}$ (藤黄酸) & $2.54 \pm 0.17$ & $1.80 \pm 0.09$ & $0.41 \pm 0.04$ \\
Doxorubicin & $0.17 \pm 0.03$ & $0.13 \pm 0.07$ & $0.14 \pm 0.01$ \\
\hline
\end{tabular}

\section{2 结论}

对藤黄酸在酸性条件下的重排反应研究发现, 藤黄 酸在单一醋酸溶剂中不发生重排反应, 在添加强质子酸 对甲苯磺酸条件下, $80{ }^{\circ} \mathrm{C}$ 条件下, $1 \sim 3 \mathrm{~h}$ 反应生成 2 种 主要的重排产物 2 和 3 . 在微波条件下, 藤黄酸在含有 对甲苯磺酸的醋酸中, $80{ }^{\circ} \mathrm{C}$ 下, 反应 $5 \mathrm{~min}$, 反应产物 仍是 $\mathbf{2}$ 和 3 , 产率更高. 通过现代波谱技术解析出两种 重排产物的结构. 重排产物 2 的结构在先前的研究论文 中被报道过 ${ }^{[8]}$, 但是由于原文仅有氢谱(氞代试剂与本 课题组使用的相同, 均为 $\mathrm{CDCl}_{3}$ ), 并且数据与我们的有 较大差距: 如文献中指出 $\mathrm{H}-4$ 化学位移在 $\delta 3.49$, 我们 
课题组得到的该氢信号在 $\delta 4.15$; 文献给出 H-24 的化学 位移在 $\delta 1.30$, 本课题组得到的该氢信号在 1.71 ; 文献 给出 H-25 信号在 $\delta 1.73$, 而本课题组得到的该氢信号在 $\delta 1.29$; 文献给出 H-36 信号在 $\delta 2.51 \sim 2.54$ 处, 与本课题 组得到的 H-36a 在 $\delta 2.39$ 和 H-36b 在 $\delta 1.69$ 有较大出入, 且该文献并未给出 H-29 的氢信号化学位移值. 我们通 过二维核磁波谱首次全归属了这一化合物. 重排产物 3 是一个新化合物. 化合物 2 和 3 都显示强于前体的细胞 毒活性，证明酸催化重排藤黄酸产物非常有意义.

\section{3 实验部分}

\section{1 仪器与试剂}

实验仪器: Nicolet 3080 型红外光谱仪( $\mathrm{KBr}$ 压片); Bruker $\mathrm{AV}-400$ 和 $500 \mathrm{MHz}$ 核磁共振仪 $\left(\mathrm{CDCl}_{3}\right.$ 为溶剂, TMS 为内标); 低分辨质谱用 Agilent 1100 LC/MSD SL 质谱仪; 高分辨用 Bruker Daltonics, Inc. APEXIII 7.0 TESLA FT 质谱仪; HPLC 用 Waters 600E, 检测器为 Waters 2489 紫外可见检测器, 检测波长为 $210,254 \mathrm{~nm}$; 分析柱为 SunFireTM-C18 column $(4.6 \mathrm{~mm} \times 150 \mathrm{~mm}, 5$ $\mu \mathrm{m})$, 半制备柱为 SunFireTM-C18 column $(10 \mu \mathrm{m} \times 250$ $\mathrm{mm}, 5 \mu \mathrm{m})$; 微波反应器为上海比朗仪器制造, 型号为 BILON-ZCW-1000W.

实验试剂: 藤黄(Gamboge)于 2010 年购于中国广 西. 冰醋酸、对甲苯磺酸皆为分析纯, 分别采购于上海 国药集团化学试剂有限公司.

\section{2 实验方法}

\subsection{1 藤黄酸制备和鉴定}

称取块状藤黄树脂 $1000 \mathrm{~g}$, 粉碎成 50 目以下, 用 $1500 \mathrm{~g}$ 的工业硅藻土与藤黄粉末充分混合均匀, 填入层 析柱用丙酮洗脱, 洗脱液近无色时停止洗脱, 将洗脱液 减压蒸馏得到藤黄粗提浸膏 $880 \mathrm{~g}$.

将 $880 \mathrm{~g}$ 藤黄粗体浸膏按溶于 $1 \mathrm{~L}$ 吡啶中, 超声将藤 黄充分溶解, 再加入吡啶体积约 $1 / 10$ 的水, 封口, 在 $4{ }^{\circ} \mathrm{C}$ 冰箱中放置 $24 \mathrm{~h}$, 抽滤, 用石油醚洗涤, 抽滤得藤 黄酸吡啶盐粗品.

将上述粗品用尽量少的甲醇在 $80{ }^{\circ} \mathrm{C}$ 条件下充分溶 解, 趁热抽滤. 将所得滤液封口, 在 $4{ }^{\circ} \mathrm{C}$ 冰箱中放置 24 $\mathrm{h}$ 后, 抽滤, 将所得固体依照上述方法重结晶 $3 \sim 4$ 次, 即可得到纯度非常高的藤黄酸吡啶盐晶体.

将上述藤黄酸吡啶盐晶体加入 $2 \mathrm{~mol} / \mathrm{L}$ 盐酸中, 剧 烈振荡 $15 \mathrm{~min}$, 然后用 $\mathrm{CH}_{2} \mathrm{Cl}_{2}$ 和饱和 $\mathrm{NaCl}$ 溶液萃取 $2 \sim 3$ 次, 取 $\mathrm{CH}_{2} \mathrm{Cl}_{2}$ 层, 无水 $\mathrm{Na}_{2} \mathrm{SO}_{4}$ 干燥, 旋蒸后得到 橙黄色的藤黄酸粉末. ${ }^{1} \mathrm{H}$ NMR $\left(400 \mathrm{MHz}, \mathrm{CDCl}_{3}\right) \delta$ : 12.74 (s, 1H, 6-OH), 7.54 (d, $J=6.8 \mathrm{~Hz}, 1 \mathrm{H}, \mathrm{H}-10), 6.56$ (d, $J=10.1 \mathrm{~Hz}, 1 \mathrm{H}, \mathrm{H}-4), 6.12$ (t, $J=7.1 \mathrm{~Hz}, 1 \mathrm{H}, \mathrm{H}-27)$, $5.34(\mathrm{~d}, J=10.1 \mathrm{~Hz}, 1 \mathrm{H}, \mathrm{H}-3), 5.03(\mathrm{~d}, J=6.9 \mathrm{~Hz}, 2 \mathrm{H}$, H-32, H-37), $3.53 \sim 3.44$ (m, 1H, H-11), 3.29 (dd, $J=14.4$, $8.2 \mathrm{~Hz}, 1 \mathrm{H}, \mathrm{Ha}-31), 3.12$ (d, $J=11.7 \mathrm{~Hz}, 1 \mathrm{H}, \mathrm{Hb}-31), 2.99$ (d, $J=7.0 \mathrm{~Hz}, 2 \mathrm{H}, \mathrm{H}-26), 2.51$ (d, $J=9.2 \mathrm{~Hz}, 1 \mathrm{H}, \mathrm{H}-22)$, 2.31 (dd, $J=13.3,4.0 \mathrm{~Hz}, 1 \mathrm{H}, \mathrm{Ha}-21), 2.00$ (d, $J=7.5 \mathrm{~Hz}$, 2H), 1.73 (s, 3H), 1.71 (s, 3H), 1.69 (s, 3H), 1.64 (s, 3H), $1.62(\mathrm{~s}, 3 \mathrm{H}), 1.54(\mathrm{~s}, 3 \mathrm{H}), 1.35(\mathrm{~s}, 3 \mathrm{H}), 1.29(\mathrm{~s}, 3 \mathrm{H})$; ESI-MS $m / z: 629[\mathrm{M}+\mathrm{H}]^{+}$; HR-ESI-MS calcd for $\mathrm{C}_{38} \mathrm{H}_{45} \mathrm{O}_{8}[\mathrm{M}+\mathrm{H}]^{+}$629.31144, found 629.31089.

\subsection{2 藤黄酸重排产物 $\mathbf{2}$ 和 $\mathbf{3}$ 的制备}

在 $250 \mathrm{~mL}$ 反应瓶中加入 $1 \mathrm{~g}$ 藤黄酸 $(1.59 \mathrm{mmol})$, $100 \mathrm{~mL}$ 冰醋酸, $4 \mathrm{~g}$ 对甲苯磺酸 $(23.3 \mathrm{mmol}), 80{ }^{\circ} \mathrm{C}$ 条件 下反应 $1 \mathrm{~h}$, 将反应液放置冷却到常温, 然后倒入 200 $\mathrm{mL}$ 冰水中, 用 $\mathrm{CH}_{2} \mathrm{Cl}_{2}$ 和饱和 $\mathrm{NaHCO}_{3}$ 洗涤 5 次将酸除 掉, 无水 $\mathrm{Na}_{2} \mathrm{SO}_{4}$ 干燥, 将萃取液蒸干, 残留物用硅胶 (200 300 目)柱层析, 洗脱液条件为 $V$ (乙酸乙酯) : $V($ 石油醚 $): V($ 甲酸 $)=1: 25: 0.05$, 得到化合物 2 和 3 混合物, 此混合物用 HPLC 进行分离, 分离条件为 $V($ 乙 腈 $): V$ (水) $: V(0.08 \%$ 三氟乙酸 $)=80 ： 18 ： 2$ (流速 3 $\mathrm{mL} / \mathrm{min}$, 紫外检测波长 $254 \mathrm{~nm}$ 条件), 保留时间分别为 78 和 $63.5 \mathrm{~min}$, 得到化合物 $20.21 \mathrm{~g}$ (收率 $21 \%$ ) 和化合物 $30.17 \mathrm{~g}$ (收率 17\%).

在 $50 \mathrm{~mL}$ 反应瓶中加入 $1 \mathrm{~g}$ 藤黄酸、 $10 \mathrm{~mL}$ 冰醋酸、 $0.4 \mathrm{~g}$ 对甲苯磺酸, 在微波反应器中 $80{ }^{\circ} \mathrm{C}$ 条件下反应 5 $\mathrm{min}$, 冷却到常温, 倒入 $20 \mathrm{~mL}$ 冰水中, 用 $\mathrm{CH}_{2} \mathrm{Cl}_{2}$ 和饱 和 $\mathrm{NaHCO}_{3}$ 洗涤 5 次将酸除掉, 无水 $\mathrm{Na}_{2} \mathrm{SO}_{4}$ 干燥, 将萃 取液蒸干, 得到粗品, 粗品用硅胶(200 300 目)柱层析, 洗脱液条件为 $V($ 乙酸乙酯 $): V($ 石油醚 $): V($ 甲酸 $)=1$ : $20: 0.05$, 得到化合物 $\mathbf{2}$ 和 $\mathbf{3}$ 的混合物, 分离后分别得到 0.44 和 $0.42 \mathrm{~g}$, 产率分别为 $44 \%$ 和 $42 \%$

39-氢-37-烯-转位藤黄酸(2): ${ }^{1} \mathrm{H}$ NMR 和 ${ }^{13} \mathrm{C}$ NMR 数据见表 $1 . \mathrm{IR}(\mathrm{KBr}) v: 3434,2965,2925,2855,1727$, 1669, 1637, 1591, 1433, 1377, 1324, 1260, 1219, 1178, 1135, 1053, $1022 \mathrm{~cm}^{-1}$; ESI-MS m/z: $629[\mathrm{M}+\mathrm{H}]^{+}$; HR-ESI-MS calcd for $\mathrm{C}_{38} \mathrm{H}_{45} \mathrm{O}_{8}[\mathrm{M}+\mathrm{H}]^{+}$629.31144, found 629.31089 .

38,39-二氢-36-烯-转位藤黄酸(3): ${ }^{1} \mathrm{H}$ NMR 和 ${ }^{13} \mathrm{C}$ NMR 数据见表 1. IR (KBr) v: 3441，2925，2855，1737， 1684, 1637, 1429, 1375, 1327, 1260, 1196, 1139, 1086, $1037 \mathrm{~cm}^{-1}$; ESI-MS m/z: $629[\mathrm{M}+\mathrm{H}]^{+}$; HR-ESI-MS calcd for $\mathrm{C}_{38} \mathrm{H}_{45} \mathrm{O}_{8}[\mathrm{M}+\mathrm{H}]^{+} \quad 629.3114$ 4, found 629.31089 .

\section{2 .3 细胞毒活性测试}

样品配制：用二甲基亚砜(DMSO) (Merck)溶解后， 加入磷酸盐缓冲液(PBS) (一)配成 $1000 \mathrm{~g} / \mathrm{mL}$ 的溶液或 
均匀的混悬液, 然后用含 DMSO 的 PBS (一)稀释. 活性 测试采用 MTT 法: 96 孔板每孔加入浓度为 $4 \sim 5 \times 10^{4}$ 个 $/ \mathrm{mL}$ 的细胞悬液 $100 \mu \mathrm{L}$, 置 $37{ }^{\circ} \mathrm{C}, 5 \% \mathrm{CO}_{2}$ 培养箱内. $24 \mathrm{~h}$ 后, 加入样品液, $10 \mu \mathrm{L} /$ 孔, 设双复孔, $37{ }^{\circ} \mathrm{C}, 5 \%$ $\mathrm{CO}_{2}$ 作用 $72 \mathrm{~h}$. 每孔加入 $5 \mathrm{mg} / \mathrm{mL}$ 的 MTT 溶液 $20 \mu \mathrm{L}$, 作用 $4 \mathrm{~h}$ 后加入溶解液, $100 \mu \mathrm{L} /$ 孔, 置培养箱内, 溶解后 用全波长多功能酶标仪测 $570 \mathrm{~nm}$ OD 值. 抑制率 $=(1-$ OD 实验组)/OD 对照组 $\times 100 \%$. 阳性对照为藤黄酸(1) 和阿霉素(Doxorubicin).

辅助材料(Supporting Information) 所合成化合物的 ${ }^{1} \mathrm{H}$ NMR, ${ }^{13} \mathrm{C}$ NMR, HSQC, HMBC, DEPT135谱图数据. 这些材料可以免费从本刊网站(http://sioc-journal.cn/)上 下载.

\section{References}

[1] Hou, W.; Xiao, W. Chin. Tradit. Herb. Drugs 2011, 42, 617 (in
Chinese).

(侯文洁，萧伟，中草药, 2011, 42, 617.)

[2] Han, Q. B.; Wang, Y. L.; Yang, L. Chem Pharm Bull. 2006, 54, 265.

[3] Lu, Y. M.; Wang, G.; Ye, D. J. J. Chin. Mater. Med. 1996, 21, 90 (in Chinese).

(陆跃鸣, 王耿, 叶定江，中国中药杂志, 1996, 21, 90.)

[4] Guo, Q. L.; Zhao, L.; You, Q. D. Chin. J. Nat. Med. 2004, 2, 106 (in Chinese).

(郭青龙, 赵丽, 尤启东, 中国天然药物, 2004, 2, 106.)

[5] Liu, J. B.; Qin, S. K.; Li, J. Chin. Clin. Oncol. 2005, 10, 472 (in Chinese).

(刘静冰, 秦叔達, 李进, 临床肿瘤学杂志, 2005, 10, 472.)

[6] Feng, F.; Liu, W. Y.; Chen, Y. S.; Guo, Q. L.; You, Q. D. J. Chin. Pharm. Univ. 2005, 36, 302 (in Chinese).

(冯峰, 柳文媛, 陈优生, 郭青龙, 尤启东, 中国药科大学学报, 2005, 36, 302.)

[7] Wang, X. J.; Lu, N.; You, Q. D.; Guo, Q. L. Bioorg. Med. Chem. Lett. 2010, 20, 2438.

[8] Wang, X. J.; Ma, J. H.; You, Q. D.; Guo, Q. L. Eur. J. Med. Chem. 2010, 45, 4343.

(Li, L.; Fan, Y.) 